5 Research Square

\title{
Circulating Glycan Monosaccharide Composite Detects Endometrial Cancer at Early Stages
}

pengjiao zeng

The Affiliated Hospital of Qingdao University

\section{Yulong Chen}

The Affiliated Hospital of Qingdao University

\section{Cui Hao}

The Affiliated Hospital of Qingdao University

\section{Xuan Zeng}

The Affiliated Hospital of Qingdao University

\section{Yanyun Gao}

The Affiliated Hospital of Qingdao University

\section{Dandan Yang}

The Affiliated Hospital of Qingdao University

\section{Ming Shan}

The Affiliated Hospital of Qingdao University

\section{Huaiqian Dou}

The Affiliated Hospital of Qingdao University

\section{Meng Zhang}

The Affiliated Hospital of Qingdao University

\section{Yiran Zhang}

The Affiliated Hospital of Qingdao University

\section{Yanli He}

The Affiliated Hospital of Qingdao University

\section{Xiulian Li}

The Affiliated Hospital of Qingdao University

\section{Juan Li}

The Affiliated Hospital of Qingdao University

\section{Liming Wang}

The Affiliated Hospital of Qingdao University

\section{Qin Yao}

The Affiliated Hospital of Qingdao University

Lijuan Zhang ( $\square$ zhanglj@qduhospital.cn )

Affiliated Hospital of Qingdao University 
Keywords: Serum, Endometrial cancer, Biomarker, Monosaccharide composite, Glycan, Pre-column derivation, High-performance liquid chromatography (HPLC).

Posted Date: July 7th, 2021

DOI: https://doi.org/10.21203/rs.3.rs-657137/v1

License: (c) (i) This work is licensed under a Creative Commons Attribution 4.0 International License. Read Full License 


\section{Abstract \\ Background}

Early diagnosis is the key to cure endometrial cancer. However, current cancer biomarkers, such as CA125, have low specificity and sensitivity for endometrial cancer diagnosis even at late stages. Glycans, containing both genetic and environmental information, are the most promising biomolecules to serve as early diagnostic biomarkers. To simplifying the structural information residing in the circulating glycans, the monosaccharide composite was investigated as a biomarker in the current study.

\section{Methods}

Acid hydrolysis, pre-column derivation, and HPLC analysis were used to quantify monosaccharide compositions of serum glycans for pathological confirmed endometrial cancer patients $(n=30)$, uterine fibroid patients $(n=35)$, and the healthy controls $(n=30)$. The levels of CA125 were also measured for all the serum samples from the patients and healthy controls. Receiver operating characteristic and logistic regression were used to assess the reliability of the biomarker.

\section{Results}

Galactosamine, glucosamine, and galactose concentrations were significantly increased $(P<0.05)$, whereas mannose and fucose concentrations were decreased (for fucose, $P<0.05$ ) in hydrolyzed serum glycans of endometrial cancer patients compared to the healthy control. The area under curve (AUC), $95 \%$ confidence interval, sensitivity, specificity, and accuracy were $0.96,0.92-1.00,86.7 \%, 93.3 \%$, and $90.0 \%$ for monosaccharide composite and $0.58,0.43-0.73,40.0 \%, 83.3 \%$, and $61.7 \%$ for CA 125 , respectively. Furthermore, the monosaccharide composite detected 28 while CA125 only detected 5 out of the 30 patients with pathologically confirmed endometrial cancer.

\section{Conclusions}

The serum monosaccharide composition analysis was a simple and quantitative assay for biomarker development. The monosaccharide composite of hydrolyzed serum glycans was an excellent biomarker for endometrial cancer early detection. The abnormal environmental information present in the circulating glycans explained its ability to detect cancers at early stages.

\section{Background}

The American Cancer Society estimates 66,620 new endometrial cancer (EC) cases will be diagnosed in 2020, and 12,590 will succumb to the disease[1]. By 2030, EC will be the third most common cancer among American women [2]. EC is diagnosed by a combination of transvaginal ultrasound scans of (TVUS) and endometrial biopsies clinically. The invasiveness and discomfort in postmenopausal women have a visual analog scale (VAS) pain score of $6.5[3,4]$. In addition, a large number of technical problems $(12-23 \%)$ and 
insufficient tissue quantity (16-68\%) in obtaining an endometrial biopsy are also reported [5]. Therefore, it is necessary to develop non-invasive blood-based biomarkers. The ideal biomarkers must accurately improve early diagnosis and outcomes, including the survival of EC patients[6]. However, there is no reliable circulating biomarker for EC detection even though CA125 is one of the most reported biomarkers for EC[7, 8].

Animal glycome is estimated to be $10^{4}$ times larger than the proteome due to the compact genetic and environmental information stored in glycan structures [9-12]. It has been well documented that total serum $O$ or $\mathrm{N}$-glycans are different among healthy individuals and patients suffering from different types of cancers [13-16]. Over 125 congenital disorders of glycosylation (CDG) discovered in humans are associated with multi-systems dysfunctions [17]. Thus, abnormal glycan structures are the common features for both inherited and environment-related diseases. In addition, different $\mathrm{N}$-glycan structures in serum IgGs that have no direct relationships with cancer cells can distinguish healthy individuals from patients with lung, gastric, prostate, ovarian, and breast cancers[18-22]. These published facts indicate cancers are systems diseases, and the diverse glycan structures, abundantly present in human blood circulation and tissues, are the most promising biomolecules for biomarker discovery [23]. However, glycan structure-based biomarkers are difficult to translate into clinical use due to the structural heterogeneity among patients with different blood types and other technical and time-consuming issues [24-26].

All clinically used cancer biomarkers established during the past 40 years are either specific glycan structures or heavily glycosylated proteins $[27,28]$. Among clinically used cancer biomarkers, CA125 is a heavily glycosylated protein, which was first identified as an ovarian cancer biomarker by hybridoma monoclonal antibody technology[29, 30]. Our recent study showed that serum CA125 levels are increased in patients suffering fibrosis-associated diseases, such as lung fibrosis, cirrhosis, and nephrotic syndrome, more than ovarian and other types of cancers[31]. These observations indicated that the limited amount of glycan information in CA125 fails to serve as a cancer biomarker.

The human serum glycan structures are difficult to characterize [27]. However, they all consist of 10 monosaccharides, i.e., glucose, Glc; galactose, Gal; glucosamine, GlcN; galactosamine, GalN; fucose, Fuc; mannose, Man; xylose, Xyl; glucuronic acid, GIcA; iduronic acid, IdoA; and 5-N-acetyIneuraminic acid or sialic acid. However, few methods have been developed to quantify monosaccharide compositions in human sera or plasmas for biomarker development. Thus, we have taken the approach by optimizing the assay over the past nine years[32]. We subsequently discovered that plasma monosaccharide compositions are different in 11 different types of cancers[33]. Using this method [34,35], we obtained the monosaccharide compositions in patients suffering from EC $(n=30)$ and uterine fibroid $(n=35)$ and in the healthy individuals $(n=30)$. We also used the serum CA125 levels as references for patients suffering EC or uterine fibroid. Our data showed that the monosaccharide composite of hydrolyzed serum glycans was a much better diagnostic biomarker over CA125 for EC early diagnosis.

\section{Methods}

\section{Patient cohort}

In this hospital-based case-control study, all participants were those who visited the Affiliated Hospital of Qingdao University between June 2015 to January 2016 (Table 1). Patients with a history of chronic infection 
diseases, abnormal kidney function, pregnancy, and neoplastic disease were excluded from the study. The medical records of $30 \mathrm{EC}$ and 35 uterine fibroid patients included in the current study were reviewed clinically and pathologically from the first onset of symptoms to hospitalization at the Department of Obstetrics and Gynecology. FIGO staging was available for all 30 EC patients. Among them, 26 were in stage I and II; 3 were in stage III, and 1 was in stage IV. Age-matched healthy individuals $(n=30)$ free from any signs or symptoms of the endometrial disease were served as healthy control. These individuals were recruited from a geographic background similar to that of the patients. Informed consent was obtained from each patient included in the study. The study protocol conforms to the ethical guidelines of the 1975 Declaration of Helsinki and was conducted following the guidelines set by the Ethics Committee of the Affiliated Hospital of Qingdao University.

\section{Sample processing}

Fasting blood samples were collected for both patients and healthy individuals, while the fasting blood from EC patients were drawn prior to surgery. The serum samples were obtained by centrifuging at $3500 \mathrm{rpm}$ for 5 minutes. The serum samples were aliquoted and stored at $-80^{\circ} \mathrm{C}$ for further analysis.

\section{CA125 measurement}

Serum CA125 levels were quantified by the Roche automatic electro-chemiluminescence immunoassay system (Roche Diagnostic $\mathrm{GmbH}$ ) according to manufacturer's instructions.

\section{Pre-column derivation by HPLC for monosaccharide composition analysis of serum samples}

The monosaccharide composition analysis was conducted by using ampule sealed acidolysis followed by PMP-labeling and HPLC analysis. The detailed experimental procedures we have optimized and used for the current study can be found in[32].

\section{Statistical analysis}

All data were analyzed with SPSS statistical software (version 23.0; SPSS Inc., Chicago, Illinois, USA), GraphPad Prism Software 8.0 (GraphPad Software Inc.,

San Diego, CA, USA). We used the multi-sample Shapiro-Wilk test to examine the normality of continuous variables. Categorical variables are presented as percentages, and continuous variables are presented as mean \pm standard deviation or median (25th percentile, 75th percentile). Use Student's t test (normal distribution) or Mann-Whitney $U$ test (skew distribution) to compare the difference of continuous variables and use the Chisquare test to compare the distribution of categorical variables. Kruskal-Wallis test (a non-parametric ANOVA) was used to compare differences between groups.

Logistic regression was used to test the interactive effects of CA125, serum monosaccharides on the observed association between the healthy and EC group. Receiver operating characteristic (ROC) curves analysis was established to evaluate the diagnostic ability of CA125 and monosaccharide composite as biomarkers. The best statistical cutoff values of serum monosaccharides were calculated. The sensitivity and specificity for selected cutoff points were then assessed. The $P \leq 0.05$ was considered statistically significant. AUC values of 
$\leq 0.5,0.5$ to $<0.7,0.7$ to $<0.8$, and 0.8 to $<0.9$ and $\geq 0.9$ indicate no, poor, acceptable, excellent and outstanding discrimination, respectively.

\section{Results}

\section{Serum CA125 levels and the six monosaccharide concentrations in hydrolyzed serum glycans were different among the control, EC, and uterine fibroid groups.}

The demographic and clinical characteristics for patients and healthy individuals are summarized in (Table 1). The average ages were $57.00 \pm 1.65$ years for EC patients, $55.69 \pm 1.14$ years for patients suffering uterine fibroid, and $57.23 \pm 1.69$ years for the healthy control group $(P=0.719)$. There were also no statistically significant differences in $B M I(P=0.060)$, menopausal status $(P=0.361)$, hypertension $(P=0.467)$, and diabetes $(P=0.792)$.

Serum CA125 levels were shown in (Fig. 1a) for the three groups. The increased median CA125 levels were observed in the uterine fibroid group compared to that in the EC, and the control groups. Uterine fibroid group had significantly increased serum CA125 levels compared to the control group $(p<0.0001)$ as well as to the EC group $(p<0.01)$, which was consistent with our previous finding that CA125 is associated with fibrosisassociated diseases more than with cancers[31].

The concentrations of six monosaccharides, including Fuc, Gal, GaIN, Glc, GlcN, and Man, in hydrolyzed serum samples of all three groups obtained by our optimized method[32] were summarized in (Fig. 1b). Median Gal and Glc concentrations were significantly increased $(P<0.05)$, whereas Fuc concentration was significantly decreased $(P<0.05)$ in hydrolyzed serum glycans of the EC group compared to the control groups.

Unexpectedly, all six monosaccharide concentrations were significantly different $(P<0.05)$ between the EC and uterine fibroid groups. Remarkably, Man, GlcN, and Gal concentrations were only significantly decreased in the uterine fibroid group compared to the control group. Moreover, Glc concentration was significantly increased $(P$ $<0.05)$ in the EC group but significantly decreased $(P<0.05)$ in the uterine fibroid group compared to the control group.

\section{Monosaccharide concentrations in hydrolyzed serum glycans were potential early diagnostic biomarkers for EC.}

Early diagnosis will be critical in preventing EC-related death. We next tested if the monosaccharide concentration changed at the early stages of EC development. Figure 2 showed significantly changes in Fuc ( $P$ $=0.001)$, GalN $(P=0.04)$, and Glc $(P=0.04)$ concentrations were present in stage $I / I$. No significant changes in six monosaccharide concentrations were observed between the EC patients at stage I/ $\nabla$ and stage $\nabla / I V$. Thus,

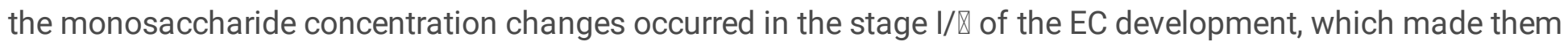
suitable for the early EC diagnosis.

\section{Monosaccharide composite of hydrolyzed serum glycans was a better biomarker than CA125 for the EC diagnosis}

We next investigated the diagnostic properties of CA125, six monosaccharides from hydrolyzed serum glycans, and the monosaccharide composite as EC biomarkers by performing the receiving operator curve (ROC) 
analysis (Fig. 3). Since all six monosaccharide concentrations were obtained and quantified in the same chromatogram of a HPLC analysis, we used logistic regression analysis to test the monosaccharide composite as biomarker for EC diagnosis. The following logistic regression model for monosaccharide composite was established:

-12.131-13.909Man-1.666GlcN + 41.358GaIN + 1.873Glc + 20.977Gal - 26.359Fuc. The detailed AUC, 95\% confidence interval $(\mathrm{Cl})$, sensitivity, specificity, accuracy, positive predicting value (PPV), negative predicting value (NPV) and $P$ values for CA125, each monosaccharide, and the logistic regression model were summarized in Table 2.

Among the monosaccharides, the AUC value for Fuc was the highest $(0.75,95 \%$ confidence interval, $0.63-0.87$, $\mathrm{P}<0.001)$. Using $0.32 \mathrm{mmol} / \mathrm{L}$ as the cutoff value, the sensitivity and specificity for Fuc as a biomarker for EC diagnosis were $56.7 \%$ and $86.7 \%$, respectively (Fig. 3), which were better than CA125 as a biomarker for EC diagnosis. The AUC for monosaccharide composite as a biomarker for EC diagnosis was further increased to 0.96 . The area under curve (AUC), $95 \%$ confidence interval, sensitivity, specificity, and accuracy were $0.96,0.92-$ $1.00,86.7 \%, 93.3 \%$, and $90.0 \%$ for monosaccharide composite biomarker and $0.58,0.43-0.73,40.0 \%, 83.3 \%$, and $61.7 \%$ for CA125, respectively (Table 2). Therefore, the monosaccharide composite had the advantage over any single monosaccharide or CA125 as a circulating biomarker for EC diagnosis.

When the cutoff value was set at 0.396 for the logistic regression model, the novel monosaccharide composite biomarker detected 28 while CA125 only detected 5 out of 30 EC patients (Fig. 4). Thus, monosaccharide composite was an excellent biomarker for EC diagnosis.

\section{Discussion}

No reliable biomarker in blood circulation for EC diagnosis is clinically available. Thus, a simple, low-cost, and non-invasive circulating monosaccharide composite-based biomarker was developed for EC diagnosis while using clinical cancer biomarker CA125 and patients suffering uterine fibroid as references. Our data demonstrated that the monosaccharide composite of hydrolyzed serum glycans was an excellent biomarker for EC diagnosis (Fig. 3 and Table 2) in that it detected 28, while the current clinically used cancer biomarker, CA125, only detected 5 out of 30 patients suffering from EC (Fig. 4). Increased fucose concentration alone in hydrolyzed serum samples of EC patients served as a better biomarker than CA125 for EC detection (AUCs: 0.75 vs.0.58, Table 2). Furthermore, CA125 was a better circulating biomarker for uterine fibroid than EC (Fig. 1), which was consistent with our previous report that CA125 is a better biomarker for fibroid-associated diseases than for cancers [31].

The molecular building blocks in blood circulation include 20 amino acids that make proteins, eight nucleotides that build up DNA and RNA, ten monosaccharides in glycans, and eight lipids[36]. Unlike RNAs and proteins, glycans and lipids are not directly encoded by DNAs but contribute to the pathogenesis and severity of an increasing number of human diseases[37, 38]. Therefore, valid plasm/serum-based cancer biomarkers could be protein/peptides, DNA, RNA, glycans, lipids, and metabolites present in blood circulation. In recent years, the "OMICS" technologies including genomics[39], epigenomics[40], proteomics[41], glycomics[42], lipidomics[43], and metabolomics[44] have been used to compare the molecular differences between the 
plasm/serum samples from healthy individuals and patients suffering cancers systematically. However, no biomarkers have been developed into clinical applications yet.

Glycans are the most information-dense and structurally dynamic biomolecules, which are structurally different in health and diseases [45]. Thus, serum glycans are the most attractive biomolecules for biomarker discovery. However, over 30 clinically used serum cancer biomarkers have relatively low sensitivity and specificity due to their limited glycan information contents $[27,46]$. Currently, nearly all studies of serum glycans as possible cancer biomarkers have been focused on resolving complicated serum glycan structures by the complicated preparation procedures plus expensive instrumentations, such as LC-MS, which makes it hard to translate such research results into clinical applications. Thus, our approach is novel to simplify the serum glycan information into quantitative monosaccharide compositions.

The bottleneck of our approach is to optimize the serum glycan hydrolysis conditions. In our previous study, we used a Picotag station to optimize acid hydrolysis conditions for glycosaminoglycans[47-49]. By using the Picotag station-based hydrolysis condition, we found that the serum GlcN and GalN concentrations in patients with lung cancers were significantly higher than that of healthy individuals, and the same assay also detects the contaminant in heparin $[47,50]$. Since no Picotag station was available in China, we optimized a workable hydrolysis condition for serum or plasma samples[32]. However, the ampule sealed acid hydrolysis used in the current study is time-consuming. Moreover, even using the optimized acidolysis condition, we found that when adding monosaccharide standards into serum, the added GlcA had the highest loss rate of $52.78 \%$, followed by Glc (23.70\%) and $\mathrm{Xyl}$ (30.94\%)[32]. Thus, further optimizing acid hydrolysis conditions is desirable for clinical applications.

Our study has several advantages for blood circulation-based biomarker development and clinical applications. First, our method includes a simple PMP-labeling procedure, a clean chromatograph with baseline separation of all monosaccharides, excellent sensitivity, and linearity that were reported 21 years ago [35]. Secondly, this method can be readily implemented in any laboratory with typical HPLC equipment. Thirdly, only $10 \mu \mathrm{l}$ of sera from either EC patients or healthy individuals were used for the assay without any other manipulation. Fourthly, the data of six monosaccharides from a serum sample were obtained simultaneously from a single HPLC profile, which made the logistic regression analysis straightforward to generate quantitative data reports for each patient. Moreover, we found that monosaccharide composites of hydrolyzed serum glycans could serve as early diagnostic biomarkers for most types of cancers that we have studied during the past[51-53]. To further understand the molecular mechanisms, we have employed tumor and nontumor mouse models to pinpoint the source of abnormal serum glycans in diseases[54,55]. We found that livers and spleens but not tumors in the mouse model were mainly responsible for the increased monosaccharide concentrations of hydrolyzed plasma glycans[55, 56].

The weakness of the study was the limited sample size of EC patients. Another limitation was that most of the EC patients in our study were in the early stage of disease development, which did not allow for more detailed correlations between monosaccharide concentration changes with the EC progression.

\section{Conclusions}


Monosaccharide composite of hydrolyzed serum glycans was an excellent biomarker for EC early detection. The monosaccharide composite could replace currently used clinical cancer biomarkers, such as CA125, or substitute complex glycan structures to serve as a quantitative circulating biomarker for EC diagnosis. The simple and reliable monosaccharide quantification method used in the current study should reduce costs and patient risks and make the non-invasive diagnosis of EC possible. However, further studies will be required to investigate if the monosaccharide composite could also serve as the long-sought prognostic and treatment effect evaluating biomarkers for EC.

\section{Abbreviations}

BMI

Body mass index; CA125:Carbohydrate antigen 125; EC:Endometrial cancer; Fuc:Fucose; Gal:Glucosamine; GaIN:Galactosamine; Glc:Glucose; GlcA:Glucuronic acid; GlcN:Glucosamine; HPLC:High-Performance Liquid Chromatography; IdoA:Iduronic acid; LC-MS:Liquid Chromatography couple Mass Spectrometry; Man:Mannose; MC:Monosaccharide composite; PMP:1-Phenyl-3-methyl-5-pyrazolone; ROC:Receiver operating characteristic; Xyl:Xylose.

\section{Declarations}

\section{Ethics approval and consent to participate}

Informed consent was obtained from each patient included in the study. The study protocol conforms to the ethical guidelines of the 1975 Declaration of Helsinki and was conducted following the guidelines set by the Ethics Committee of the Affiliated Hospital of Qingdao University.

\section{Consent for publication}

All authors approved the manuscript and this submission.

\section{Availability of data and materials}

All human sera used in the current study are available on request under a standard material transfer agreement with the Affiliated Hospital of Qingdao University for academic research purposes only. All data generated or analyzed during this study are included in this article or associated with supplementary information files. Raw data files are available upon request.

\section{Competing interests}

The Affiliated Hospital of Qingdao University has filed patent applications based on this work.

\section{Funding}

The study was sponsored by the Natural Science Foundation of China (Grant 81672585) and the Taishan Scholar Fellowship to L.Z.

\section{Authors'contributions}


Lijuan Zhang and Qin Yao conceived and guided the research project. Pengjiao Zeng carried out the experiments. All authors participated in the sample collections, data analysis, and data interpretation. Lijuan Zhang, Pengjiao Zeng, and Yulong Chen wrote the manuscript.

\section{Acknowledgements}

This work was supported by the Department of Laboratory and Gynecology in The Affiliated Hospital of Qingdao University, for the blood sample collection and the gynecologist provides clinical diagnosis.

\section{Authors' information}

${ }^{1}$ Systems Biology \& Medicine Center for Complex Diseases, Center for Clinical Research, the Affiliated Hospital of Qingdao University, Qingdao, 266003, China, ${ }^{2}$ Department of Obstetrics and Gynecology, the Affiliated Hospital of Qingdao University, Qingdao, 266003, China, Biological Resource Center, ${ }^{3}$ the Second Affiliated Hospital of Guangzhou University of Chinese Medicine, Guangzhou区 510120, China, ${ }^{4}$ School of Pharmacy, Binzhou Medical University, Yantai, 264003, China, ${ }^{5}$ Management Center of Medical Record, the Affiliated Hospital of Qingdao University, Qingdao, 266003, China

\section{References}

1. Siegel RL, Miller KD, Jemal A, Cancer statistics. 2020. CA Cancer J Clin. 2020;70:7-30.

2. Tarney CM, Wang G, Bateman NW,Conrads KA,Zhou M, Hood BL, et al. Biomarker panel for early detection of endometrial cancer in the Prostate, Lung, Colorectal, and Ovarian cancer screening trial. Am J Obstet Gynecol. 2019;221:472. e471-472 e410.

3. Leclair CM. Zia JK,Doom CM,Morgan TK,Edelman AB. Pain Experienced Using Two Different Methods of Endometrial Biopsy A Randomized Controlled Trial. Obstetrics Gynecology. 2011;117:636-41.

4. Van Den Bosch T, Verguts J, Daemen A,Gevaert O,Domali E, Claerhout F, et al. Pain experienced during transvaginal ultrasound, saline contrast sonohysterography, hysteroscopy and office sampling: a comparative study. Ultrasound Obst Gyn. 2008;31:346-51.

5. Visser NCM, Breijer MC, Herman MC, Bekkers RLM,Veersema S, Opmeer BC, et al. Factors attributing to the failure of endometrial sampling in women with postmenopausal bleeding. Acta Obstet Gyn Scan. 2013;92:1216-22.

6. Donkers H, Bekkers R, Galaal K. Diagnostic value of microRNA panel in endometrial cancer: A systematic review. Oncotarget. 2020;11:2010-23.

7. Jiang T, Huang L, Zhang S. Preoperative serum CA125: a useful marker for surgical management of endometrial cancer. BMC Cancer. 2015;15:396.

8. Bian J, Sun X, Li B, Ming L. Clinical Significance of Serum HE4, CA125, CA724, and CA19-9 in Patients With Endometrial Cancer. Technol Cancer Res Treat. 2017;16:435-9.

9. Cummings RD, Pierce JM. The challenge and promise of glycomics. Chem Biol. 2014;21:1-15.

10. Zhang L. Glycosaminoglycans in development, health and disease. Preface Progress in molecular biology translational science. 2010;93:xvii-xviii. 
11. Schachter H, Freeze HH. Glycosylation diseases: quo vadis? Biochimica et biophysica acta. 2009;1792:925-930.

12. Hart GW, Copeland RJ. Glycomics hits the big time. Cell. 2010;143:672-6.

13. He Y, Xie Q,Wang Y, Liang Y,Xu X, Li Y, et al. Liquid chromatography mass spectrometry-based O-glycomics to evaluate glycosylation alterations in gastric cancer. Proteomics Clin Appl. 2016;10:206-15.

14. Hatakeyama S, Amano M, Tobisawa Y,Yoneyama T,Tsuchiya N, Habuchi T, et al. Serum N-glycan alteration associated with renal cell carcinoma detected by high throughput glycan analysis. J Urol. 2014;191:80513.

15. Kim JH, Park CW,Um D,Baek KH,Jo Y, An H, et al. Mass spectrometric screening of ovarian cancer with serum glycans. Dis Markers. 2014;2014:634289.

16. Nouso K, Amano M, Ito YM,Miyahara K,Morimoto Y, Kato H, et al. Clinical utility of high-throughput glycome analysis in patients with pancreatic cancer. Journal of gastroenterology. 2013;48:1171-9.

17. Ng BG, Freeze HH. Perspectives on Glycosylation and Its Congenital Disorders. Trends Genet. 2018;34:466-76.

18. Kodar K, Stadlmann J, Klaamas K, Sergeyev B,Kurtenkov O. Immunoglobulin G Fc N-glycan profiling in patients with gastric cancer by LC-ESI-MS: relation to tumor progression and survival. Glycoconj J. 2012;29:57-66.

19. Kanoh Y, Ohara T,Mashiko T,Abe T,Masuda N,Akahoshi T. Relationship between N-linked oligosaccharide chains of human serum immunoglobulin $\mathrm{G}$ and serum tumor markers with non-small cell lung cancer progression. Anticancer Res. 2006;26:4293-7.

20. Kanoh Y, Mashiko T,Danbara M, Takayama Y, Ohtani S, Egawa S, et al. Changes in serum IgG oligosaccharide chains with prostate cancer progression. Anticancer Res. 2004;24:3135-9.

21. Kawaguchi-Sakita N, Kaneshiro-Nakagawa K, Kawashima M, Sugimoto M, Tokiwa M, Suzuki E, et al. Serum immunoglobulin G Fc region N-glycosylation profiling by matrix-assisted laser desorption/ionization mass spectrometry can distinguish breast cancer patients from cancer-free controls. Biochem Biophys Res Commun. 2016;469:1140-5.

22. Saldova R, Royle L, Radcliffe CM, Abd Hamid UM, Evans R, Arnold JN, et al. Ovarian cancer is associated with changes in glycosylation in both acute-phase proteins and IgG. Glycobiology. 2007;17:1344-56.

23. Lan Y, Hao C, Zeng X, He YL,Zeng PJ, Guo ZH, et al. Serum glycoprotein-derived N- and O-linked glycans as cancer biomarkers. Am J Cancer Res. 2016;6:2390-415.

24. Jensen PH, Karlsson NG, Kolarich D,Packer NH. Structural analysis of N- and O-glycans released from glycoproteins. Nat Protoc. 2012;7:1299-310.

25. Messina A, Palmigiano A,Esposito F,Fiumara A,BordugoA, Barone R, et al. HILIC-UPLC-MS for high throughput and isomeric $\mathrm{N}$-glycan separation and characterization in Congenital Disorders Glycosylation and human diseases. Glycoconj J. 2020.

26. Kolarich D, Jensen PH,Altmann F, Packer NH. Determination of site-specific glycan heterogeneity on glycoproteins. Nat Protoc. 2012;7:1285-98.

27. Hu M, Lan Y, Lu AMa X,Zhang L. Glycan-based biomarkers for diagnosis of cancers and other diseases: Past, present, and future. Prog Mol Biol Transl Sci. 2019;162:1-24. 
28. Zhang L. Glycans and Glycosaminoglycans as Clinical Biomarkers. Preface. Progress in molecular biology and translational science. 2019;163:xvii-xviii.

29. Kohler G, Milstein C. Continuous cultures of fused cells secreting antibody of predefined specificity. Nature. 1975;256:495-7.

30. Bast RC, Jr.,Klug TL,St John E, Jenison E,Niloff JM, Lazarus H, et al. A radioimmunoassay using a monoclonal antibody to monitor the course of epithelial ovarian cancer. The New England journal of medicine. 1983;309:883-887.

31. Zhang M, Zhang YFu J,Zhang L. Serum CA125 levels are decreased in rectal cancer but increased in fibrosis-associated diseases and in most types of cancers. Prog Mol Biol Transl Sci. 2019;162:241-52.

32. Zeng P, Zeng X,Hao C,Zhang Y, Zhang M, Zhang L. Ampule-sealed Acidolysis for Monosaccharide Composition Analysis of Serum or Plasma Samples. Nature protocol exchange. 2020.

33. Zhang $L$, Zeng $X$,Lan Y,Qiu P,Zeng $Y$, Xu L, et al., inventorsApplication of a method for detecting monosaccharides hydrolyzed from blood samples for cancer detection. China2015.

34. Fu D, O'Neill RA. Monosaccharide composition analysis of oligosaccharides and glycoproteins by highperformance liquid chromatography. Anal Biochem. 1995;227:377-84.

35. Honda S, Akao E,Suzuki S,Okuda M, Kakehi K,Nakamura J. High-performance liquid chromatography of reducing carbohydrates as strongly ultraviolet-absorbing and electrochemically sensitive 1-phenyl-3methyl-5-pyrazolone derivatives. Anal Biochem. 1989;180:351-7.

36. Marth JD. A unified vision of the building blocks of life. Nat Cell Biol. 2008;10:1015-6.

37. Fahy E, Subramaniam S, Murphy RC,Nishijima M, Raetz CR, Shimizu T, et al. Update of the LIPID MAPS comprehensive classification system for lipids. J Lipid Res. 2009;50 Suppl:S9-14.

38. Ohtsubo K, Marth JD. Glycosylation in cellular mechanisms of health and disease. Cell. 2006;126:855-67.

39. Stephens PJ, Tarpey PS, Davies H, Van Loo P, Greenman C, Wedge DC, et al. The landscape of cancer genes and mutational processes in breast cancer. Nature. 2012;486:400-4.

40. Sturm D, Witt H, Hovestadt V, Khuong-Quang DA, Jones DT, Konermann C, et al. Hotspot mutations in H3F3A and IDH1 define distinct epigenetic and biological subgroups of glioblastoma. Cancer cell. 2012;22:425-37.

41. Rohlff C. New approaches towards integrated proteomic databases and depositories. Expert Rev Proteomics. 2004;1:267-74.

42. Vanderschaeghe D. Festjens N,Delanghe J,Callewaert N. Glycome profiling using modern glycomics technology: technical aspects and applications. Biological chemistry. 2010;391:149-61.

43. Han X. An update on lipidomics: progress and application in biomarker and drug development. Curr Opin Mol Ther. 2007;9:586-91.

44. Wang X, Zhang A,Sun H. Power of metabolomics in diagnosis and biomarker discovery of hepatocellular carcinoma. Hepatology. 2013;57:2072-7.

45. Zhang L. Glycosaminoglycan (GAG) biosynthesis and GAG-binding proteins. Prog Mol Biol Transl Sci. 2010;93:1-17.

46. Tang Y, Cui Y, Zhang S, Zhang L. The sensitivity and specificity of serum glycan-based biomarkers for cancer detection. Prog Mol Biol Transl Sci. 2019;162:121-40. 
47. Pan J, Qian Y, Weiser P,Zhou X,Lu H, Studelska DR, et al. Glycosaminoglycans and activated contact system in cancer patient plasmas. Prog Mol Biol Transl Sci. 2010;93:473-95.

48. Studelska DR, Giljum K,McDowell LM,Zhang LJ. Quantification of glycosaminoglycans by reversed-phase HPLC separation of fluorescent isoindole derivatives. Glycobiology. 2006;16:65-72.

49. McDowell LM, Frazier BA,Studelska DR,Giljum K, Chen J, Liu J, et al. Inhibition or activation of Apert syndrome FGFR2 (S252W) signaling by specific glycosaminoglycans. J Biol Chem. 2006;281:6924-30.

50. Pan J, Qian Y, Zhou XD,Pazandak A,Frazier SB, Weiser P, et al. Oversulfated chondroitin sulfate is not the sole contaminant in heparin. Nat Biotechnol. 2010;28:203-7.

51. Hao C, Zhang Q, Su W,Xuan Y, Zeng X, Qiu T, et al. Circulating Monosaccharide Composite-based Glycan Biomarker Diagnoses Non-small Cell Lung Cancer at Early Stages. Journal of Thoracic Oncology. 2021; Submitted.

52. He Y, Gao P, Li X, Zhang Y, Zhang M, Dou H, et al. Monosaccharide Composite of Hydrolyzed Serum Glycans is a reliable Biomarker for Early Diagnosis of Kidney Cancer. Kidney International. 2020;Submitted.

53. Wang X, Huang X,He YZhang Y, Hao C, Zeng P, et al. Circulating Free and Glycan Monosaccharide Composite-based Biomarkers Diagnose Colorectal Cancer at Early Stages and Predicts the Curing Rate after Surgical Resection. Gut. 2020;Submitted.

54. Ian Y,Hao, Wang C, Li X,He G, Zhang Y. M, et al. Reduced glycocalyx content explains Bleomycin-induced lung fibrosis. American journal of respiratory and critical care medicine. 2021;Submitted..

55. Lan Y, Wang X, Zeng X, Li X, Zeng P, Liu Y, et al. Increased circulating glycan contents reflect systems' reactions to tumors and body's cancer belligerent capability. Gut. 2020.

\section{Tables}

Due to technical limitations, table 1 is only available as a download in the Supplemental Files section. 
Table 2

CA125, six monosaccharides, and monosaccharide composite (MC) as biomarkers for EC diagnosis. Area under the receiver operating characteristic curve (AUC), 95\% confidence interval (CI), sensitivity, specificity, positive predictable values (PPV), negative predictable values (NPV), accuracy, and P values of CA125, six monosaccharides, and monosaccharide composite as diagnostic biomarkers for EC.

\begin{tabular}{|c|c|c|c|c|c|c|c|c|}
\hline Variables & AUC & $95 \% \mathrm{CL}$ & Sensitivity/\% & Specificity/\% & PPV/\% & NPV/\% & Accuracy/\% & $\begin{array}{l}P \\
\text { value }\end{array}$ \\
\hline CA125 & 0.58 & $\begin{array}{l}0.43- \\
0.73\end{array}$ & 40.0 & 83.3 & 70.0 & 41.9 & 61.7 & 0.25 \\
\hline Fuc & 0.75 & $\begin{array}{l}0.63- \\
0.87\end{array}$ & 56.7 & 86.7 & 77.3 & 65.8 & 70.0 & $\begin{array}{l}< \\
0.001\end{array}$ \\
\hline Gal & 0.55 & $\begin{array}{l}0.40- \\
0.70\end{array}$ & 46.7 & 76.7 & 69.6 & 37.8 & 65.0 & 0.49 \\
\hline GalN & 0.68 & $\begin{array}{l}0.54- \\
0.81\end{array}$ & 90.0 & 40.0 & 61.4 & 81.3 & 66.7 & $<0.05$ \\
\hline Glc & 0.65 & $\begin{array}{l}0.51- \\
0.79\end{array}$ & 66.7 & 63.3 & 54.1 & 56.5 & 55.0 & $<0.05$ \\
\hline GlcN & 0.51 & $\begin{array}{l}0.37- \\
0.66\end{array}$ & 20.0 & 93.3 & 66.7 & 47.1 & 55.0 & 0.80 \\
\hline Man & 0.57 & $\begin{array}{l}0.43- \\
0.72\end{array}$ & 36.4 & 83.0 & 68.8 & 56.8 & 60.0 & 0.30 \\
\hline MC & 0.96 & $\begin{array}{l}0.92- \\
1.00\end{array}$ & 86.7 & 93.3 & 92.9 & 87.5 & 90.0 & $\begin{array}{l}< \\
0.0001\end{array}$ \\
\hline
\end{tabular}

Figures 
a

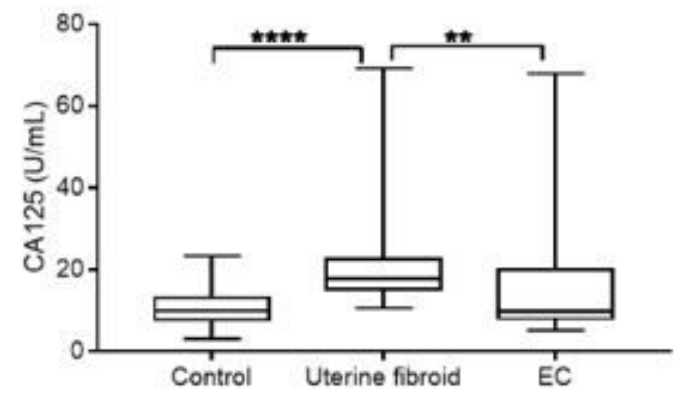

b
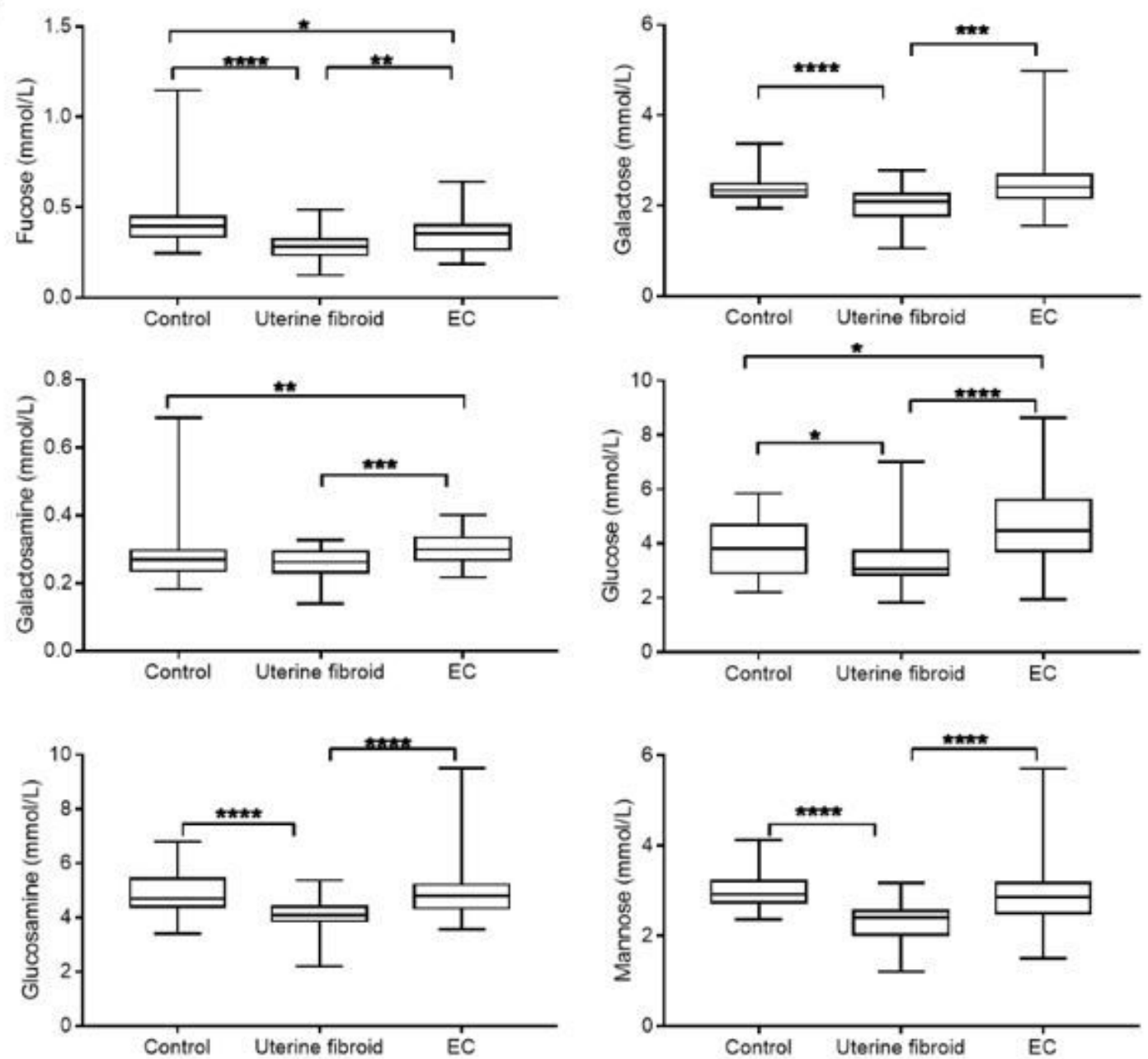

Figure 1

Serum CA125 levels and monosaccharides concentrations were different among control and uterine fibroid groups. a. Serum CA125 levels. b. Six monosaccharide concentrations in the hydrolyzed serum glycans. The lower $(25 \%)$, median ( $50 \%)$, and upper ( $75 \%$ ) quartiles as well as $95 \%$ confident intervals $(2.5 \%$ to $97.5 \%)$ were marked for $\operatorname{EC}(n=30)$, uterine fibroid( $(n=35)$, and control $(n=30)$. ****: $p \leq 0.0001, * * *: p \leq 0.001, * *: p \leq 0.01$, **: $p \leq 0.01$. Kruskal-Wallis test (a non-parametric ANOVA)) was used to compare differences between groups. 
a

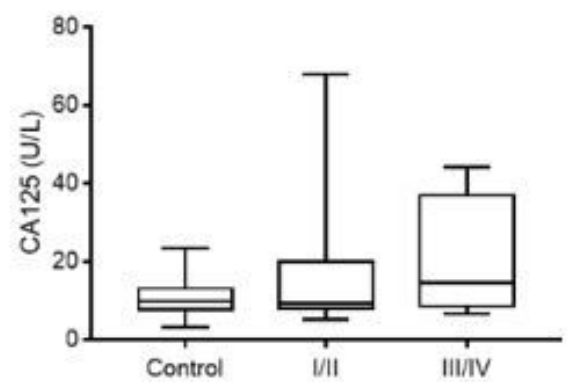

b
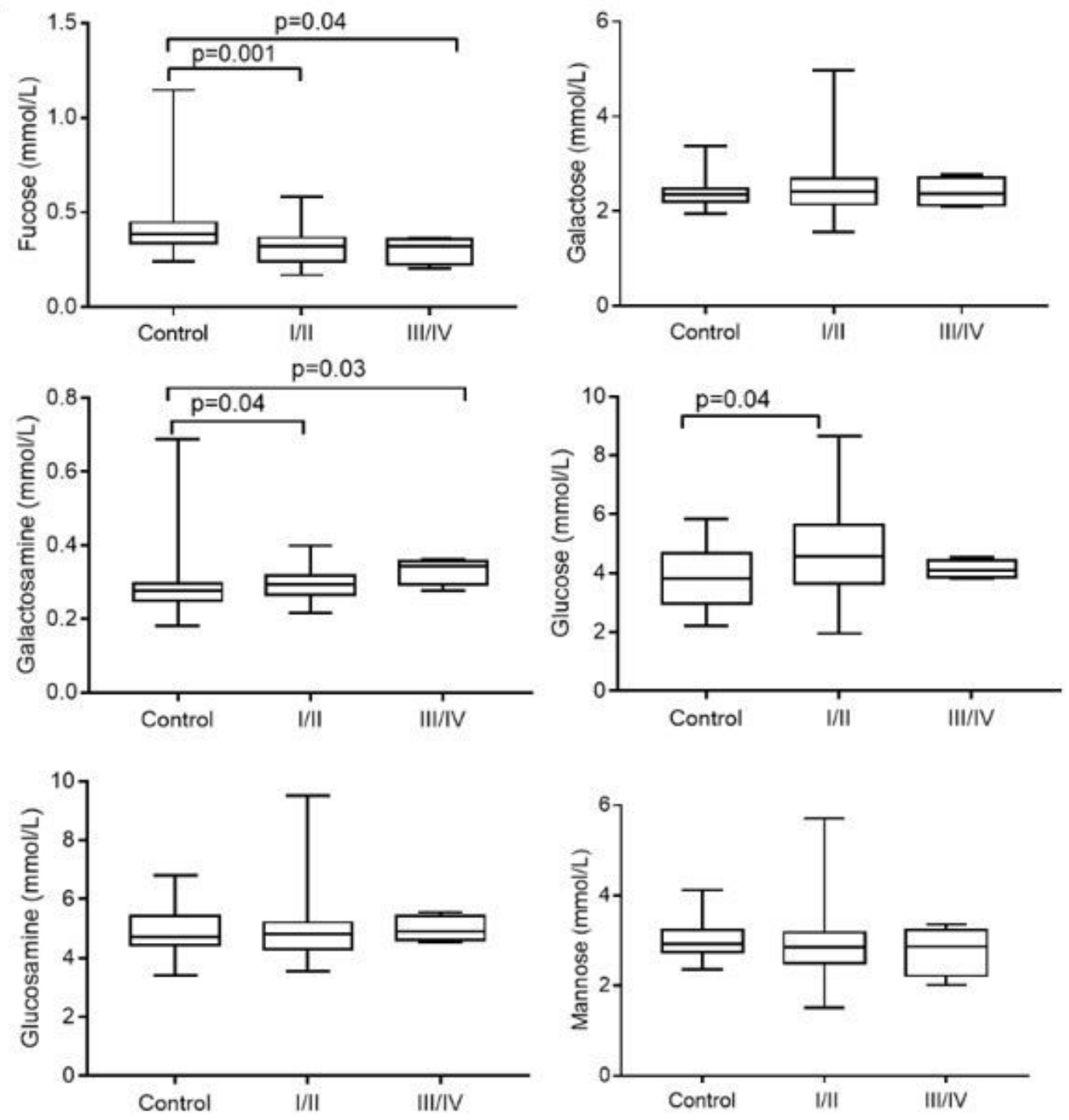

\section{Figure 2}

Serum monosaccharides concentrations of EC patients at early and late stages were different from controls. The lower (25\%), median (50\%), and upper ( $75 \%$ ) quartiles as well as $95 \%$ confident intervals $(2.5 \%$ to $97.5 \%)$ were marked for EC patients at stages I/II vs. III/IV in contrast to control. Groups were compared using the Kruskal-Wallis test (a non-parametric one-way ANOVA). 
a

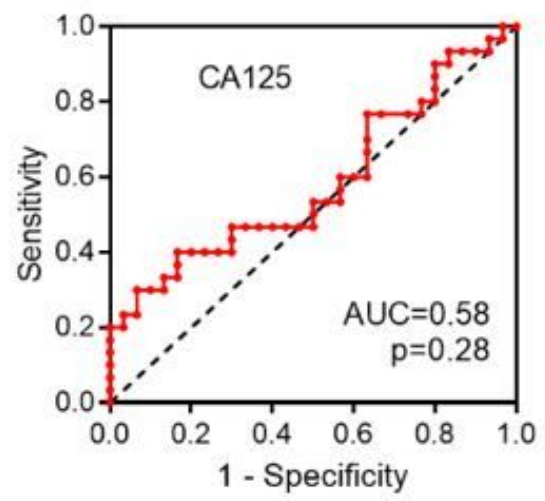

b
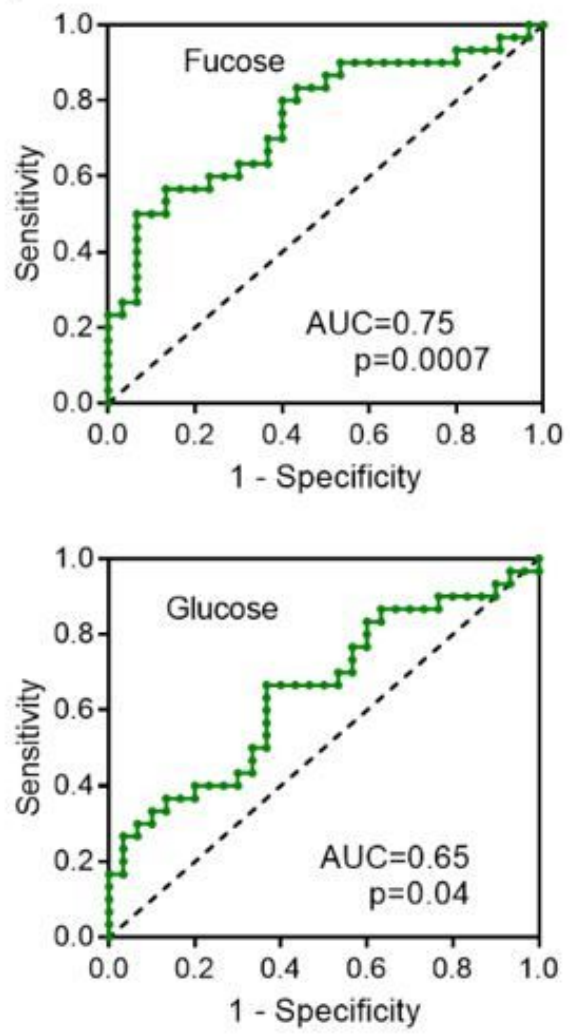

c
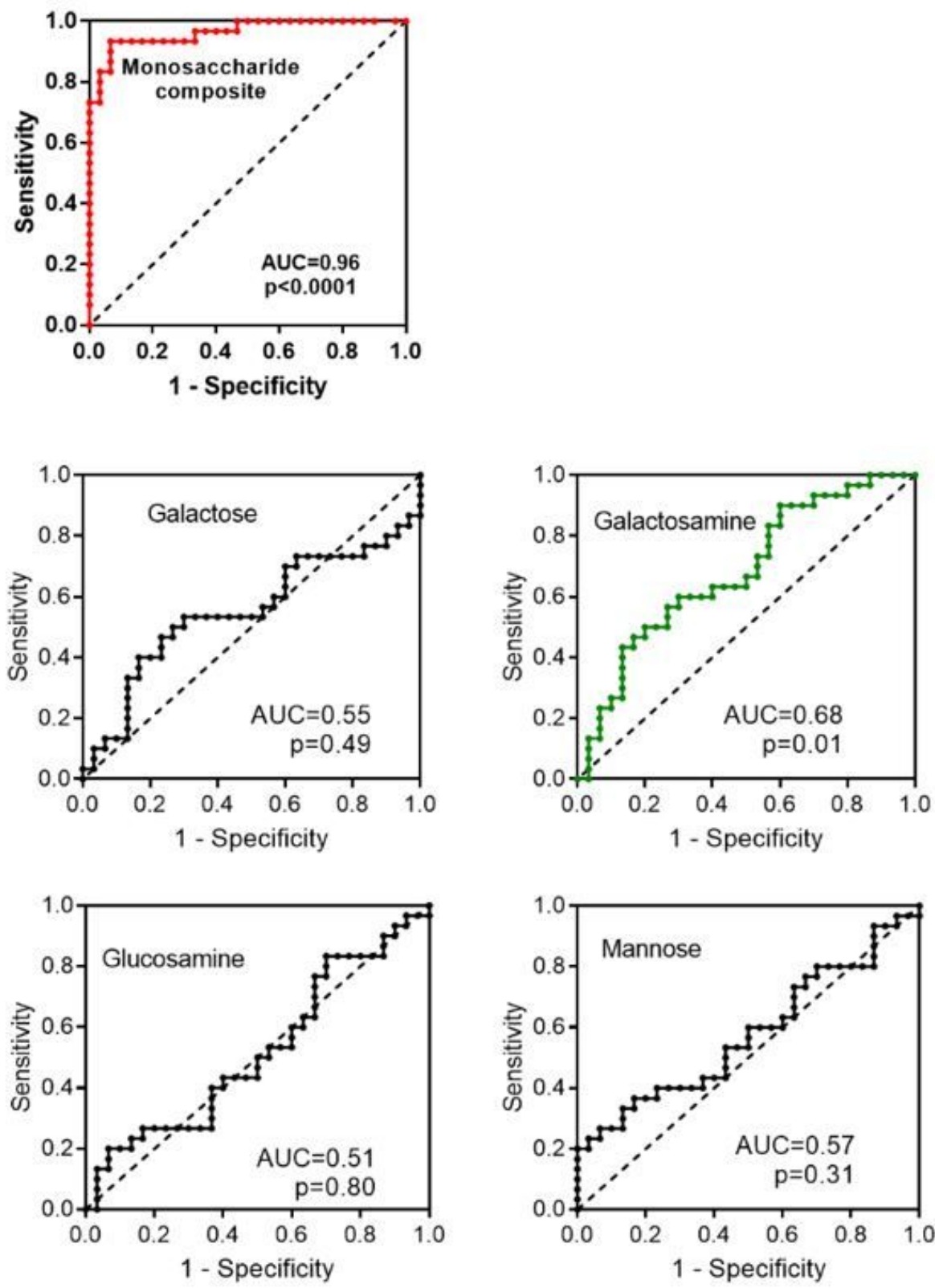

\section{Figure 3}

Monosaccharide composite was better biomarker than CA125 for EC diagnosis assessed by ROC curve analysis. a. CA125 (AUC=0.58); b. Six monosaccharides (AUC=0.75 for Fuc). C. Monosaccharides composite $(A U C=0.96)$. 


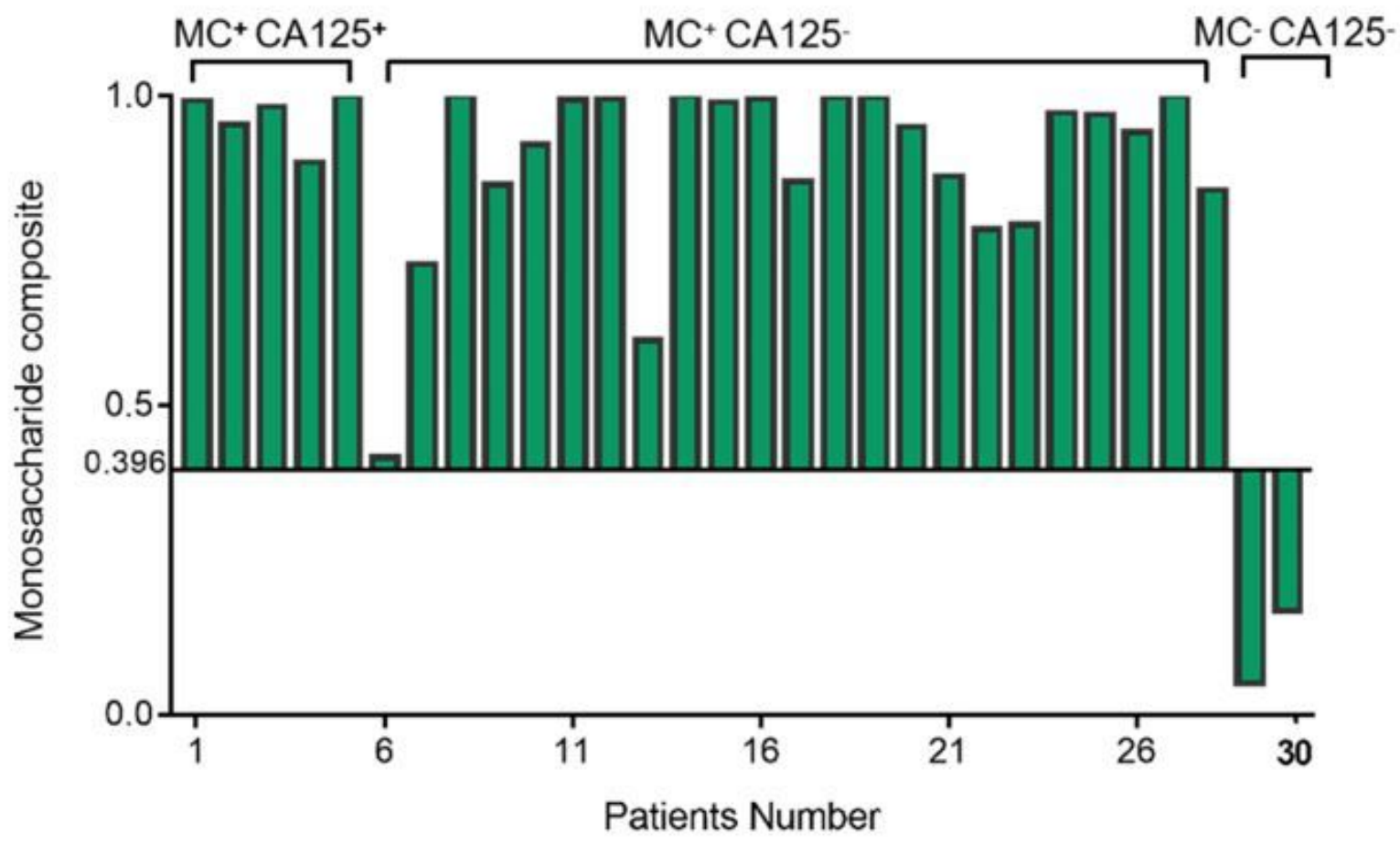

Figure 4

Monosaccharide composite (MC) was an excellent biomarker for EC diagnosis. Among 30 pathologically confirmed EC patients, 5 patients were positive for both MC and CA125, 23 patients were positive for MC and negative for $\mathrm{CA} 125$, and 2 patients were negative for both $\mathrm{MC}$ and $\mathrm{CA} 125$.

\section{Supplementary Files}

This is a list of supplementary files associated with this preprint. Click to download.

- Table1.jpg 